\title{
How can hospital administrators prevent the seasonal flu among hospital staff?
}

\author{
Yumi Jo ${ }^{1}$, Masahiro Hirose ${ }^{2}$, Toshihiko Kawamura ${ }^{3}$, Chiaki Sano ${ }^{3}$, Shunichi Kumakura ${ }^{3}$, \\ and Junji Suzumiya ${ }^{3}$ \\ ${ }^{1}$ Shimane University Hospital \\ ${ }^{2}$ Shimane University Faculty of Medicine Graduate School of Medicine \\ ${ }^{3}$ Affiliation not available
}

July 2, 2020

\begin{abstract}
Objectives: Reducing influenza infection among hospital employees is vital to prevent the spread of this infection among patients. A questionnaire study was performed to investigate seasonal influenza infection and vaccination among hospital employees, with the aim of decreasing the incidence of influenza. Methods: From 2014 to 2017, we distributed a questionnaire to hospital employees who were eligible for vaccination. The following information was collected: age of respondents, whether they lived with children aged $<15$ years, their preseason vaccination status, and their history of seasonal influenza infection. The data were analyzed and averaged for all 4 influenza seasons. Results: The incidence of influenza was $6.2 \%$ among employees through 4 seasons. The inoculation rate of the influenza vaccine ranged from $92 \%$ to $99 \%$. The odds ratio for influenza infection among employees living with children aged $<15$ years was $2.19(\mathrm{P}<0.0001)$. A decrease in influenza infection was not observed in vaccinated employees $(\mathrm{P}=0.75)$. Conclusions: The results suggested that influenza infection in hospital personnel is not due to a low vaccination rate. Among people living with children younger than 15 years, the odds ratio of influenza infection was $2.19(\mathrm{P}<0.0001)$. These findings suggest that protection against influenza infection at home is particularly required for hospital employees living with children aged $<15$ years.
\end{abstract}

\section{Introduction}

Hospital employees often come into contact with patients, children, and older adults. Annual influenza vaccination is recommended for staff to avoid contracting this disease and spreading it to others, especially hospital patients. According to the WHO fact sheet, the "seasonal flu vaccine is the most effective method of preventing infection."

The influenza vaccine has been reported to have protective efficacy for healthy adults even if the vaccine differs from the subtype of the prevalent virus. Furthermore, vaccination decreases the risk of complications and death among older adults, children, and patients with underlying diseases, despite inadequate prevention [1-5]. In Japan, vaccinations are provided once or twice a year for each applicant.

According to the home page of the Ministry of Health, Labor and Welfare, the influenza vaccine does not entirely prevent infection but has a potent effect on aggravation of the disease. However, the reported decrease in the prevalence of influenza infection due to vaccination varies among studies [6-10]. Recently, an increase in immunocompromised patients and the spread of new influenza virus strains have led to an upsurge in the vaccination rate. Reports have indicated that high-dose vaccination or two-dose vaccination can be useful $[11,12]$. However, it became difficult to obtain sufficient vaccines for outpatients and the general population in 2017 , thus the supply of vaccines available is insufficient to employ such methods. 
Moreover, hospital employees still take time off because of influenza infection despite undergoing vaccination, and this adversely impacts their regular duties. In our hospital, prophylactic medication is prescribed at the hospital's expense for staff members who come into frequent contact with patients with influenza without taking infection precautions. However, these measures are not common in Japan. Preventive measures were given to 78 staff members at the hospital's expense in the 2015/2016 season. In the 2016/2017 season, preventive measured were given to 158 staff members at the hospital's expense. The aim of this observational study was to investigate factors associated with influenza infection and measures that can reduce the impact of influenza on hospital personnel.

\section{Methods}

\section{Data collection}

Hospital employees participating in the hospital program for seasonal influenza vaccination were enrolled and received a questionnaire. Seasonal flu is prevalent from December until April of the next year. In our hospital, the vaccine is administered starting on October 30 and ending by the second week in November. The questionnaire was distributed within two days before the start date of vaccine administration and collected with a preliminary examination sheet on the day of the inoculation.

This questionnaire collected information on the age of each respondent, whether the individual lived with children aged $<15$ years, whether the person had been vaccinated in the preceding fiscal year (preseason vaccination status), and the respondent's history of seasonal influenza infection in the preceding fiscal year. We asked people who answered "I had flu" the following additional question: Was your diagnosis of influenza infection based on the result of an influenza diagnosis kit? The number of vaccinated personnel was determined by the number of injected ampoules. In this study, one season ranged from September to August of the next year, and "2017" refers to the period from September 2016 to August 2017. Only coded information that was anonymized was used.

\section{Statistical analysis}

The statistical analysis was performed with JMP14.2@ (SAS Institute Japan). In this study, the answers to questions were classified based on whether or not participants were infected with seasonal flu, and the two groups were compared. We considered people diagnosed with influenza using a frozen section diagnosis kit as infected with influenza in this study. When a diagnosis of influenza was involved in the clinical course or family infection only, the answer was excluded. If an item in the questionnaire was not answered, it was excluded. Between-group comparisons were performed with the chi-square test. We calculated odds ratios for the risk of developing influenza, and possible factors predicting infection were determined by logistic regression analysis. The level of significance was set at $P<0.05$.

This study was a case-control study and was not designed to evaluate a vaccine effect.

\section{Ethics statement}

This study was approved by the Medical Ethics Committee at Shimane University Faculty of Medicine.

\section{Results}

\section{Basic data}

The number of subjects who were vaccinated and the number of vaccines administered are shown in Table 1. In our hospital, we assume the staff of outside manufacturer working inside the hospital subjects of the vaccination, and an accurate number of subjects inoculated during the vaccination period could not be determined.

The vaccination rate was $92 \%$ in 2015 . However, the vaccination rate was higher than $95 \%$ in other years. In particular, physicians of the faculty of medicine and the hospital personnel are sometimes transferred to or from other institutions. Therefore, we do not have an accurate count of the number of staff members when 
vaccines were administered. For reference, we included the number of hospital personnel as of April 1 for each year.

The number of collected questionnaires is shown in Table 1 . The response rates were approximately $65 \%$ in $2014,79 \%$ in $2015,86 \%$ in 2016 , and $83 \%$ in 2017 . The collection rate increased annually. After 2015 , the effective answer rate was approximately $76 \%$.

Table 2 shows the characteristics of the study population.

\section{Prevalence of seasonal influenza}

The seasonal flu prevalence during the entire period was $6.2 \%$ (Table 3 ). The prevalence was $5.3 \%$ in 2014 , $4.9 \%$ in $2015,6.7 \%$ in 2016 , and $7.5 \%$ in 2017 .

The gender-related prevalence rates were significantly different (Table 3 ). The annual prevalence rates in 2014-2017 were $4.7 \%$ for men and $8.6 \%$ for women $(P=0.06) .4 .0 \%$ in men and $5.3 \%$ in women $(P=0.34)$, $5.2 \%$ in men and $7.2 \%$ in women $(P=0.18)$, and $5.7 \%$ in men and $8.1 \%$ in women, $(P=0.15)$, respectively, and no significant differences were observed between genders. However, the total infection rate of women for all 4 seasons was significantly higher $(P=0.03)$ (Table 3$)$.

The prevalence of influenza infection was high among subjects in their $30 \mathrm{~s}$ in all years, with rates of $6.6 \%$ in $2014,7.9 \%$ in $2015,12.1 \%$ in 2016 , and $9.6 \%$ in 2017 . A significant difference in the distribution of infected subjects was found according to age (Table 3 ).

No difference was found in the prevalence according to occupation (Table 2).

\section{Vaccination}

Over 4 seasons, the infection rate was $6.2 \%$ in the vaccinated respondents (Vaccination+) and $5.8 \%$ in the unvaccinated respondents (Vaccination-) (Table 3). The Vaccination- group did not have a tendency for a higher infection rate $(P=0.75)$ (Table 3$)$. The annual infection prevalence rates in $2014-2017$ were $5.3 \%$ in the Vaccination+ and $5.4 \%$ in the Vaccination- group $(P=0.99), 4.9 \%$ in the Vaccination + and $4.9 \%$ in the Vaccination- group $(P=1.00), 6.8 \%$ in the Vaccination + and $5.1 \%$ in the Vaccination- group $(P=0.57)$, and $7.5 \%$ in the Vaccination+ and $8.7 \%$ in the Vaccination- group $(P=0.67)$, respectively, and no significant differences were observed between the groups.

The prevalence of seasonal influenza was not necessarily lower among vaccinated subjects than among subjects who were not vaccinated. No marked decrease or increase was observed in the prevalence of influenza infection among vaccinated subjects during each season.

\section{Living with/without children younger than 15 years old}

Over 4 seasons, the infection rate was higher in respondents living with children aged $<15$ years old (Children + ) than in those not living with children aged $<15$ years old (Children-) $(P<0.0001)$ (Table 3$)$. During 2015-2017, the infection rates were markedly higher in the Children+ group than in the Children- group.

The annual infection prevalence rates in 2014-2017 were $7.0 \%$ in the Children+ and $4.5 \%$ in the Childrengroup, with no significant difference $(P=0.09), 7.8 \%$ in the Children + and $3.3 \%$ in the Children- group $(P$ $<0.001), 10.5 \%$ in the Children+ and $4.4 \%$ in the Children- group $(P<0.001)$, and $11.1 \%$ in the Children+ and $5.7 \%$ in the Children- group $(P=0.001)$, respectively.

\section{Vaccination and living with children}

Over 4 seasons, the infection rate in the in Children+ and Vaccination+ group was $9.1 \%$, and the rate in the Children+ and Vaccination- group was $8.9 \%$, with no significant difference $(P=0.95)$. The annual infection prevalence rates in 2014-2017 were $7.3 \%$ in the Children+ and Vaccination+ group and $2.8 \%$ in the Children+ and Vaccination- group $(P=0.31), 7.6 \%$ in the Children+ and Vaccination+ group and $11.1 \%$ in the Children+ and Vaccination- group $(P=0.50), 10.3 \%$ in the Children+ and Vaccination+ group and $13.0 \%$ in the Children+ and Vaccination- group $(P=0.68)$, and $11.2 \%$ in the Children+ and Vaccination+ group 
and $11.5 \%$ in the Children+ and Vaccination- group $(P=0.96)$, respectively, and no significant differences were observed between the groups. A decrease in the prevalence due to vaccination was not observed.

Over 4 seasons, the infection rate was $4.4 \%$ in Children- and Vaccination+ group and $4.2 \%$ in Childrenand Vaccination- group, with no significant difference $(P=0.85)$. The annual prevalence rates in 2014-2017 were $4.5 \%$ in the Children- and Vaccination+ group and $5.8 \%$ in the Children- and Vaccination- group $(P$ $=0.61), 3.4 \%$ in the Children- and Vaccination + group and $1.9 \%$ in the Children- and Vaccination- group $(P$ $=0.56), 4.8 \%$ in the Children- and Vaccination+ group and $0.0 \%$ in the Children- and Vaccination- group $(P=0.14)$, and $5.5 \%$ in the Children- and Vaccination+ group and $8.2 \%$ in the Children- and Vaccinationgroup $(P=0.44)$, respectively, and no significant differences were observed between groups. In the Childrengroup, no significant difference was observed a decrease in the prevalence due to vaccination.

\section{Discussion}

Influenza vaccine inoculation costs approximately 4,000 yen in Japan. The mean salary of health care industry workers is approximately 300,000 yen according to data from the Statistics Bureau of Japan. According to the Ministry of Health, Labor and Welfare website, the influenza vaccination rate for the 2016/17season was approximately $50 \%$ for each age group from 20 years old to 64 years old. Influenza vaccine inoculation in Japan showed favorable increases until 2008, and the rate became approximately constant in 2014 after having increased by approximately $10 \%$ and then subsequently slightly increasing. According to National Hospital Organization clinical indicators in 2009, the staff influenza vaccination rate was $87.3 \%$ in 2008 . Meanwhile, the vaccination rate of hospital personnel during the 2016/17season was $92.3 \%$ in the United States [13].

The vaccination rate in our hospital was $92 \%$ in 2015 and was more than $95 \%$ after 2016 . The vaccination rate in our hospital is predicted to be sufficient. People resistant to influenza, due to the environment or biological factors, may not require vaccination. The reason for resistance cannot be determined from this study.

This is the first report showing that hospital personnel living with children exhibited a statistically significant increase in influenza infection.

The prevalence of infection in females was significantly higher than that in males. According to the comments on the questionnaire, females tend to not want to be separated from their children.

Improved precautions against infection at home may contribute more to the reduction of seasonal influenza among medical staff than taking precautions against infection in the workplace, and it is important to reduce the influenza virus exposure at home to avoid spreading this infection in the workplace. In other words, in order to control in-hospital infection, hospital employees need to be educated about prevention at home. Nagao et al. reported that the prophylactic administration of oseltamivir ( $75 \mathrm{mg} /$ day for 4 days) could prevent hospital employees from developing influenza after contact with patients with influenza [14]. The drug cost is a problem, but prophylaxis against exposure at home may be effective.

In addition to vaccination, precautions against droplet contact and hand hygiene should be promoted during the influenza season. Separating family members with influenza and healthy family members at home and avoiding eating together may be effective measures to prevent influenza. It should be considered whether vaccination of children could reduce the rate of influenza infection in hospital personnel. Furthermore, it is necessary for employees with fevers and other infection-related symptoms, even if they are mild, to stay home to prevent the spread of infection.

One report showed that the prevalence of influenza infection is reduced by vaccinating employees and that vaccination reduces sick leave [15]. However, we could not evaluate the reduction in sick leave in the present questionnaire study; therefore, further investigation is necessary.

\section{Limitation}


This study has several limitations. We examined influenza infection factors over 4 seasons. However, the effects of the vaccine might be different each season. We cannot evaluate differences in the effect of the influenza vaccine on hospital personnel and individuals who do not work in the hospital. Second, we were not able to accurately determine the number of subjects present when the questionnaire was administered. Therefore, the vaccination rate may not be accurate. Third, precautions taken against infection vary in each family. Therefore, it is difficult to conclude that all the people living with children are at increased risk of influenza infection.

Finally, frozen section diagnosis was not performed in all individuals with fever. A prospective study is necessary to evaluate an effect of the vaccine.

\section{Conclusion}

For people living with children younger than 15 years, the odds ratio of influenza infection was 2.19. Observance of the infection precautions in the home may be effective in reducing seasonal flu prevalence among hospital personnel. In other words, prevention of influenza by vaccination is necessary, but it is also important to reduce exposure to the virus.

\section{Acknowledgments}

The authors thank all of the respondents who completed the questionnaire.

The authors thank Sakane K. and Ishitobi E. for help with the vaccinations.

\section{Authors' contributions}

Conceived of and designed the experiments: YJ, CS, and SK. Performed the experiments: YJ and MH. Analyzed the data: YJ and MH. Wrote the paper: YJ, MH and JS.

\section{Funding}

None.

\section{Conflicts of Interest}

None.

\section{References}

[1] Wilhelm M. Influenza in older patients: a call to action and recent updates for vaccinations. Am J Manag Care 2018; 24: S15-24.

[2] Jefferson T, Rivetti A, Di Pietrantonj C, Demicheli V. Vaccines for preventing influenza in healthy children. Cochrane Database Syst Rev 2018; 2:CD004879. https://www.cochrane.org/CD004879/ARI_vaccinespreventing-influenza-healthy-children. pdf Accessed May 12, 2020 [3] Dos Santos G, Tahrat H, BekkatBerkani R. Immunogenicity, safety, and effectiveness of seasonal influenza vaccination in patients with diabetes mellitus: A systematic review. Hum Vaccin Immunother 2018; 14: 1853-66.

[4] Bosaeed M, Kumar D. Seasonal influenza vaccine in immunocompromised persons. Hum Vaccin Immunother 2018; 14:1311-22.

[5] Bitterman R, Eliakim-Raz N, Vinograd I, Zalmanovici Trestioreanu A, Leibovici,L, Paul M. Influenza vaccines in immunosuppressed adults with cancer. Cochrane Database Syst Rev 2018; 2:CD008983. https://www.cochranelibrary.com/cdsr/doi/10.1002/14651858.CD008983.pub3/full

[6] Rondy M, Kissling E, Emborg HD, Gherasim A, Pebody R, Trebbien R et al. Interim 2017/18 influenza seasonal vaccine effectiveness: combined results from five European studies. Euro Surveill 2018; 23: 18-00086. https://www.eurosurveillance.org/content/10.2807/1560-7917.ES.2018.23.9.18-00086 
[7] Flannery B, Chung JR, Belongia EA, McLean HQ, Gaglani M, Murthy K, Zimmerman RK, et al. Interim estimates of 2017-18 seasonal influenza vaccine effectiveness-United States. MMWR Morb Mortal Wkly Rep 2018; $67:$ 180-5.

[8] Chiu SS, Kwan MYW, Feng S, Wong JSC, Leung CW, Chan ELY, et al. Interim estimate of influenza vaccine effectiveness in hospitalised children, Hong Kong, 2017/18. Euro Surveill 2018; 23: 18-00062. https://www.eurosurveillance.org/content/10.2807/1560-7917.ES.2018.23.8.18-00062

[9] Mira-Iglesias A, López-Labrador FX, Guglieri-López B, Tortajada-Girbés M, Baselga-Moreno V, Cano $\mathrm{L}$, et al. Influenza vaccine effectiveness in preventing hospitalization of individuals 60 years of age and over with laboratory-confirmed influenza, Valencia Region, Spain, influenza season 2016/17. Euro Surveill 2018; 23: 17-00318. https://www.eurosurveillance.org/content/10.2807/1560-7917.ES.2018.23.8.17-00318

[10] Ishikane M, Kamiya H, Kawabata K, Higashihara M, Sugihara M, Tabuchi A, Kuwabara M, et al. Seasonal influenza vaccine (A/New York/39/2012) effectiveness against influenza A virus of health care workers in a long term care facility attached with the hospital, Japan, 2014/15: A cohort study. J Infect Chemother 2016; 22: 777-9.

[11] Young-Xu Y, Van Aalst R, Mahmud SM, Rothman KJ, Snider JT, Westreich D, et al. Relative vaccine effectiveness of high-dose versus standard-dose influenza vaccines among Veterans Health Administration patients. J Infect Dis 2018; 217: 1718-27.

[12] Cordero E, Roca-Oporto C, Bulnes-Ramos A, Aydillo T, Gavalda J, Moreno A, et al. Two doses of inactivated influenza vaccine improve immune response in solid organ transplant recipients: Results of TRANSGRIPE 1-2, a randomized controlled clinical trial. Clin Infect Dis 2017; 64: 829-38.

[13] Black CL, Yue X, Ball SW, Fink R, de Perio MA, Laney AS, et al. Influenza Vaccination Coverage Among Health Care Personnel- United States, 2016-17 Influenza Season. Morbidity and Mortality Weekly Report 2017; 66: 1009-15.

[14] Nagao S, Onishi M, Nishiyama C. Study of the use of oseltamivir in the prevention and treatment of 2009/H1N1 influenza. Jpn J of Infect Prevent and Cont 2012; 27: 178-82. https://doi.org/10.4058/jsei.27.178

[15] Susa R, Fuse K, Ishizawa M, Tsukada H Gejyo F. Effectiveness of influenza vaccine in hospital workers. Jpn J of Infect Prevent and Cont 2001; 16: 303-8. https://doi.org/10.11550/jsei1986.16.303

\section{Tables}

\section{Legends}

Table 1. The number of the subjects vaccinated, number of vaccines administered and questionnaire collection rate

Table 2. Characteristics of the questionnaire respondents

Children (+), respondents living with children aged $<15$ years old; Children (-), respondents not living with children aged $<15$ years old; Vaccination $(+)$, vaccinated respondents; Vaccination (-), nonvaccinated respondents

Table 3. Number and percentage of individuals with seasonal influenza among hospital staff by vaccination status, gender and whether individuals were living with children, age: relative risk and odds ratio.

Shimane Japan, September 1, 2013-August 30, 2017

Vaccination $(+)$, vaccinated respondents; Vaccination $(-)$, nonvaccinated respondents; Children $(+)$, respondents living with children aged $<15$ years old; Children (-), respondents not living with children aged $<15$ years old; RR, risk ratio; OR, odds ratio; 95\% CI, 95\% confidence interval

\section{Hosted file}


Y Joh Table 1.2.docx available at https://authorea.com/users/339245/articles/465473-how-canhospital-administrators-prevent-the-seasonal-flu-among-hospital-staff

\section{Hosted file}

Y Joh Table 3.docx available at https://authorea.com/users/339245/articles/465473-how-canhospital-administrators-prevent-the-seasonal-flu-among-hospital-staff 